Research paper

\title{
Opposing roles of inter- $\alpha$-trypsin inhibitor heavy chain 4 in recurrent pregnancy loss
}

\author{
Lan Li ${ }^{\text {a,b }}$, Bum-Chae Choi ${ }^{c}$, Ji Eun Ryoo ${ }^{\text {d }}$, Sang-Jin Song ${ }^{c}$, Chang-Zhu Pei ${ }^{\text {b }}$, Kwang Yul Lee ${ }^{\text {e }}$, \\ Jinyoung Paek ${ }^{\mathrm{f}}$, Kwang-Hyun Baek ${ }^{\mathrm{b}, *}$
}

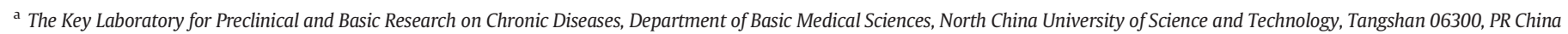

${ }^{\mathrm{b}}$ Department of Biomedical Science, CHA University, CHA General Hospital, Gyeonggi-Do 463-400, Republic of Korea

c Department of Obstetrics and Gynecology, CL Women's Hospital, Gwangju 502-800, Republic of Korea

d Department of Pharmacy, Yonsei University, Seoul 120-780, Republic of Korea

e College of Pharmacy, Research Institute of Drug Development, Chonnam National University, Gwangju 500-757, Republic of Korea

${ }^{\mathrm{f}}$ Department of Laboratory Medicine, CHA University, CHA General Hospital, Seoul 135-081, Republic of Korea

\section{A R T I C L E I N F O}

\section{Article history:}

Received 24 July 2018

Received in revised form 9 October 2018

Accepted 10 October 2018

Available online 19 October 2018

\section{Keywords:}

Early pregnancy loss

Immune tolerance

Migration and invasion

ITI-H4

Plasma kallikrein

\begin{abstract}
A B S T R A C T
Background: The mechanism behind an increased risk of recurrent pregnancy loss (RPL) remains largely unknown. In our previous study, we identified that inter- $\alpha$-trypsin inhibitor heavy chain 4 (ITI-H4) is highly expressed at a modified molecular weight of $36 \mathrm{kDa}$ in serum derived from RPL patients. Yet, the precise molecular mechanism and pathways by which the short form of ITI-H4 carries out its function remain obscure. Methods: Human sera and peripheral blood mononucleated cells (PBMCs) were collected from patients and normal controls to compare the expression levels of ITI-H4 and plasma kallikrein (KLKB1). Flow cytometric assay was performed to measure inflammatory markers in sera and culture supernatants. Furthermore, to investigate the functions of the two isoforms of ITI-H4, we performed migration, invasion, and proliferation assays. Findings: In the current study, we showed that ITI-H4 as a biomarker of RPL could be regulated by KLKB1 through the IL-6 signaling cascade, indicating a novel regulatory system for inflammation in RPL. In addition, our study indicates that the two isoforms of ITI-H4 possess opposing functions on immune response, trophoblast invasion, and monocytes migration or proliferation.

Interpretation: The ITI-H4 $\left(\Delta \mathrm{N}^{688}\right)$ might be a crucial inflammatory factor which contributes to the pathogenesis of RPL. Moreover, it is expected that this study would give some insights into potential functional mechanisms underlying RPL.

Fund: This study was supported by the Ministry of Health \& Welfare of the Republic of Korea (HI18C0378) through the Korea Health Industry Development Institute.
\end{abstract}

(c) 2018 The Authors. Published by Elsevier B.V. This is an open access article under the CC BY-NC-ND license (http://creativecommons.org/licenses/by-nc-nd/4.0/).

\section{Introduction}

Recurrent pregnancy loss (RPL) is a reproductive disorder which takes place in $2-5 \%$ of pregnancy women [1,2]. At present, increasing lines of evidence support the notion that RPL is a proinflammatory condition, and several research papers showed that pregnancy is a wellcontrolled systemic inflammatory condition [3-6]. A successful pregnancy requires strict temporal regulation of maternal immune function to accommodate the 'foreign' semi-allergenic fetus [7]. In addition, immunological causes for pregnancy loss include a problem within the

\footnotetext{
* Corresponding author at: Department of Biomedical Science, College of Life Sciences, CHA University, CHA General Hospital, 335 Pangyo-Ro, Bundang-Gu, Seongnam-Si, Gyeonggi-Do 13488, Republic of Korea.

E-mail address: baek@cha.ac.kr (K.-H. Baek).
}

embryo such that the signals to the maternal immune cells are inappropriate or a problem within the maternal immune cells [8-11]. T cells during normal pregnancy predominantly secrete anti-inflammatory cytokines (Th2 response) compared with increased pro-inflammatory cytokines (Th1 response) observed in patients with RPL, and the ratio of Th1 activity to Th2 activity is critical for normal pregnancy [10,11].

In a previous study, we used blood samples to identify altered protein expression in RPL patients undergoing in vitro fertilization (IVF) treatment [12]. One of these proteins, inter- $\alpha$-trypsin inhibitor heavy chain 4 (ITI-H4), was weakly expressed at a molecular weight of $120 \mathrm{kDa}$, but was highly expressed at a modified molecular weight of $36 \mathrm{kDa}$ in RPL patients. ITI-H4 is one of acute phase proteins, and the high expression of ITI-H4 in early liver development and explants treated with IL-6 suggests an important role in liver formation [13]. It was demonstrated that the IL-6 signaling pathway plays important 


\section{Research in Context}

\section{Evidence before this study}

Recurrent pregnancy loss (RPL) is defined as three or more consecutive miscarriages, and it affects $2-5 \%$ of couples. Often, it has been seen in patients suffering from RPL without knowing the cause. Up to now, numerous studies have been conducted to identify predictive factors that lead to RPL, nevertheless, the exact molecular mechanism underlying RPL are still not well understood yet. Over the past 15 years, our group has investigated the molecular mechanisms underlying $\mathrm{RPL}$, and we identified and reported that the cleaved form of inter- $\alpha$ trypsin inhibitor-heavy chain 4 (ITI-H4) is expressed in a large proportion of RPL patients.

\section{Added value of this study}

Here, we expand our understanding and demonstrate that ITI-H4, a key factor for RPL, could be modulated by plasma kallikrein (KLKB1) through the IL-6 signaling network, and the present study points to the opposing roles of the long isoform of ITI-H4 and ITI-H4 $\left(\Delta \mathrm{N}^{688}\right)$ in modulating inflammatory tolerance, cell invasion, migration, and proliferation.

\section{Implications of all the available evidence}

Taken all together, these results suggest that the ITI-H4 $\left(\Delta \mathrm{N}^{688}\right)$ might be a crucial inflammatory factor which contributes to the pathogenesis of RPL. This is the first study on a novel cellular mechanism underlying RPL.

roles in the regulation of diverse development and biological processes [14]. Furthermore, the high level of STAT3 expression in the luminal epithelium and stromal cells during pre-implantation period might be essential for establishing uterine receptivity [15]. ITI-H4 is the only member of the ITI family which harbors a kallikrein-released bradykinin-like domain in its C-terminal sequence, making its plasma kallikrein sensitive [16]. The cleaved form detected in the various subjects with enhanced estrogen levels may contribute to increased cleavage of ITI-H4 by elevating levels of circulating kallikrein in the serum [16]. In addition, plasma kallikrein is known to be expressed in hormone-dependent tissues such as the breast and ovary [17]. To date, the function of ITI-H4 via IL-6 signaling pathway in RPL-linked inflammatory is yet to be reported. More importantly, its action and molecular mechanism requires characterization.

In this study, we observed significantly upregulated ITI-H4 $\left(\Delta \mathrm{N}^{688}\right)$ in serum derived from RPL patients using large-scale samples. We evaluated the expression of ITI-H4 and plasma kallikrein (KLKB1) in the PBMCs of RPL patients and controls, and explored the molecular mechanism of ITI-H4's action in the development of inflammation. Our data demonstrate that inflammatory stimuli also upregulated the ITI-H4 $\left(\Delta \mathrm{N}^{688}\right)$ and KLKB1 expression in vitro. Here, we suggest that the long isoform of ITI-H4 and ITI-H4 ( $\left.\Delta \mathrm{N}^{688}\right)$ could perform opposing functions during the maintenance of pregnancy. In addition, KLKB1 might contain essential regulatory functions on this process via IL-6 signaling pathway.

\section{Materials and methods}

\subsection{Subjects and samples}

This study was approved by the Ethics Committee of CHA General Hospital located in Seoul, Korea. Informed written consents were obtained from all participants and this study with human blood samples was approved by an Institutional Review Board (Reference Number: 08-16). Subjects with regular menstrual cycles were selected and blood samples were collected from them on the $5-9^{\text {th }}$ days after ovulation of the menstrual cycle. Venous blood samples were prepared as previously described [12]. Human peripheral blood mononucleated cells (PBMC) were collected from patients who visited the Fertility Center of the CHA General Hospital in Korea. The control subjects were selected from women with no obstetric complications or history of miscarriage (Table 1). Information on the abortion history for each RPL patient is listed in Supplemental Table 1.

\subsection{Cell culture}

JEG-3 cells (placental choriocarcinoma cell line) were grown in Dulbecco's modified Eagle's medium (DMEM, GIBCO BRL, Rockville, MD, USA), and PBMC (peripheral blood mononuclear cell) and THP1 (acute monocytic leukemia cell line, ATCC TIB-202) were grown in RPMI 1640 medium supplemented with 10\% FBS (fetal bovine serum, GIBCO BRL) and $1 \%$ penicillin and streptomycin (GIBCO BRL), respectively. The cells were grown at $37^{\circ} \mathrm{C}$ in the presence of $5 \%$ carbon dioxide in a humidified incubator.

\subsection{Construction of expression vectors}

Isolation of the full-length cDNA for ITI-H4 from HepG2 cells (human hepatoma, ATCC HB-8065) was performed and the cDNA coding sequence of human ITI-H4 in GenBank (accession no. NM_002218) was subcloned into an expression vector. pCS4-3Flag vector expressing ITI$\mathrm{H} 4$ was constructed by cloning the long and $\Delta \mathrm{N}^{688}$ isoforms of PCR product of human ITI-H4 using nPfu-Forte DNA Polymerase (Enzynomics, Daejeon, Korea). KLKB1 was subcloned into pcDNA36myc expression vector. Expression constructs of ITI-H4 are as following: pCS4-3Flag-long ITI-H4, aa 1-902; pCS4-3Flag-ITI-H4 $\left(\Delta \mathrm{N}^{688}\right)$, aa 689-902; and pCS4-3Flag-ITI-H4 ( $\triangle$ BKD), $\Delta$ aa 661-688.

\subsection{Isolation of human monocyte-derived macrophages}

Peripheral blood mononuclear cells (PBMCs) from healthy women (6-8 samples for each experiment) at CHA General Hospital were isolated by Ficoll density gradient centrifugation and purified with human CD14+ MicroBeads (MiltenyiBiotec, BergischGladbach, Germany) according to the manufacturer's instructions. Monocytes were cultured in RPMI 1640 supplemented with $10 \%$ fetal calf serum for 7-10 days until differentiation into macrophages.

\subsection{Flow cytometric assay of cytokine expression}

The concentrations of cytokines in the supernatants of the treated cell cultures were measured with the BD Cytometric Bead Array

Table 1

Clinical and biochemical profiles of 30 normal controls and 60 patients with recurrent pregnancy loss (RPL).

\begin{tabular}{llll}
\hline Characteristics & Control $(\mathrm{n}=30)$ & RPL $(\mathrm{n}=60)$ & $p$-value \\
\hline Age $(\mathrm{y})$ & $31.9 \pm 4.5$ & $32.6 \pm 5.2$ & $\mathrm{NS}$ \\
BMI $\left(\mathrm{kg} / \mathrm{m}^{2}\right)$ & $24.8 \pm 2.5$ & $26.3 \pm 1.7$ & $\mathrm{NS}$ \\
Gravidity (n) & $2.5 \pm 1.7$ & $5.3 \pm 2.6$ & $<.005^{* *}$ \\
Parity (n) & $1.8 \pm 0.6$ & $1.4 \pm 0.2$ & $<.05^{*}$ \\
Previous pregnancy losses (wk) & - & $7.8 \pm 1.5$ & - \\
Spontaneous abortion (no.) & $0 \pm 0$ & $3.6 \pm 0.8$ & $<.05^{*}$ \\
No. of Primary RPL (n) & - & 42 & - \\
No. of Secondary RPL (n) & - & 18 & - \\
\hline
\end{tabular}

Numerical data were presented as a means \pm standard deviation (SD) or n; NS not significant. Abbreviations: BMI, Body mass index

$* p<.05$.

** $p<.01$ 
Human Th1/Th2/Th17 Inflammatory Cytokines Kit (BD Biosciences, San Jose, CA, USA), according to the manufacturer's instructions.

\subsection{KLKB1 activity assay}

Serum samples $(5 \mu \mathrm{L})$ were added in duplicate to $50 \mu \mathrm{L}$ of the H-ProPhe-Arg-AMC (Bachem, Bubendorf, Liestal, Switzerland) substrate with activity buffer ( $50 \mathrm{mM}$ Tris, $250 \mathrm{mM} \mathrm{NaCl}$, PH 7.5). After a $5 \mathrm{~min} 25^{\circ} \mathrm{C}$ incubation, fluorescence was recorded (excitation $380 \mathrm{~nm}$; emission $460 \mathrm{~nm}$ ) using a Flexstation 3 microplate reader. This assay was validated by the addition of $2.5 \mu \mathrm{g}$ of human plasma extracted KLKB1 (Calbiochem-Merch, Nottingham, UK) to $50 \mu \mathrm{L}$ of the substrate in activity buffer.

\subsection{Western blot analysis}

Cells were lysed in a lysis buffer (150 mM NaCl, 50 Mm Tris-HCL [pH 7.4], 1 mM PMSF, 1 mM DTT, 1\% NP-40 and protease inhibitor cocktail (PIC) tablet (Roche, Mannheim, Germany) in PBS. The primary antibodies were applied according to the provided recommendations: $\mathrm{p}$ STAT3 (Tyr705), p-STAT3 (Ser727), pERK1/2 (Thr202/Tyr204), pJNK, STAT3, JNK, and ERK1/2 antibodies (Cell Signaling Technology Inc., Danvers, MA, USA). Blots were developed by the chemiluminescent substrate using X-ray film. Intensity of bands on the Western blots was quantified by Carestream MI software and the quantitative analyses of each gray numerical value of target protein vs that of individual $\beta$ actin (Santa Cruz Biotechnology, Santa Cruz, CA, USA) or tubulin (Santa Cruz Biotechnology, Santa Cruz, CA, USA) were performed. The relative phosphorylation of target proteins was calculated by the ratios of phosphorylated proteins to $\beta$-actin and target proteins to $\beta$-actin.

\subsection{Immunoprecipitation}

JEG3 and THP1 cells were lysed and immunoprecipitated with either an anti-ITI-H4 or an anti-KLKB1 antibody (Santa Cruz Biotechnology, Santa Cruz, CA, USA) and immunoblotted with respective antibodies for detecting endogenous interaction between ITI-H4 and KLKB1. To identify the interaction between ITI-H4 and KLKB1, JEG3 cells were transfected with expression vectors encoding pCS4-3Flag-long ITI-H4, aa 1-902; pCS4-3Flag-ITI-H4 $\left(\Delta \mathrm{N}^{688}\right)$, aa 689-902; and pCS4-3FlagITI-H4 ( $\triangle$ BKD), $\Delta$ aa 661-688. The cells were cultured for $48 \mathrm{~h}$. The harvested cells were lysed with a lysis buffer ( $50 \mathrm{mM}$ Tris, pH 7.6, $150 \mathrm{mM}$ $\mathrm{NaCl}, 1$ mM EDTA, 1\% Triton X-100) supplemented with protease inhibitor cocktail (Roche Diagnostics, Mannheim, Germany). Cell lysates were incubated with the corresponding antibody $(2 \mu \mathrm{g})$ at $4{ }^{\circ} \mathrm{C}$ overnight then incubated with $30 \mu \mathrm{L}$ of protein A/G PLUS Agarose (Santa Cruz Biotechnology, Santa Cruz, CA, USA) at $4{ }^{\circ} \mathrm{C}$ for $1 \mathrm{~h}$. The samples were washed with a lysis buffer and resuspended in SDS samples buffer, and co-immunoprecipitated proteins were detected using Western blot analysis. The antibodies used for immunoblotting are anti-ITI-H4, antiKLKB1, anti-Flag (Sigma, St, Louis, MO, USA), and anti-Myc antibodies (9E10, Santa Cruz Biotechnology, Santa Cruz, CA, USA).

\subsection{Immunofluorescence microscopy}

JEG3 cells were seeded at $5 \times 10^{3}$ per well into a 12 -well culture dish (SPL Lifesciences, Gyeonggi-do, Korea), and cells were treated with or without IL-6 (10 ng/mL or $100 \mathrm{ng} / \mathrm{mL}$ ). Cell staining procedure was followed as described previously [18]. Cell images were captured by using Nikon Eclipse 50i microscopy (Nikon, Melville, NY, USA).

\subsection{RT-PCR and quantitative real-time PCR ( $q R T-P C R)$}

RNAs from cells were extracted using the TRIzol ${ }^{\mathrm{TM}}$ (Invitrogen, Carlsbad, CA, USA). $2 \mu \mathrm{g}$ total RNA for each sample was reverse-transcribed using the Oligo dT primer by Superscript ${ }^{\mathrm{TM}}$ II (Invitrogen, Carlsbad, CA,
USA). RT-PCR was performed using the AccuPower ${ }^{\mathrm{TM}}$ PCR PreMix (Bioneer, Daejeon, Korea). The PCR conditions were as follows: initial melting $\left(94{ }^{\circ} \mathrm{C}, 2 \mathrm{~min}\right), 35-40$ cycles of amplification $\left(94{ }^{\circ} \mathrm{C}\right.$, $\left.20 \mathrm{~s} / 45-65{ }^{\circ} \mathrm{C}, 30 \mathrm{~s} / 72{ }^{\circ} \mathrm{C}, 30 \mathrm{~s}\right)$, and final extension $\left(72{ }^{\circ} \mathrm{C}, 3 \mathrm{~min}\right)$. Concentration of each cDNA was normalized using the RT-PCR bands of Gapdh. qRT-PCR was performed using the SYBR® Green PCR Master Mix (Applied Biosystems, CA, USA), according to the manufacturer's instructions, in the ABI 7500 Real-time PCR System. All qRT-PCR results were normalized to Gapdh and $\beta$-actin levels. The primer sequences of the genes tested are shown in Supplemental Table 2.

\subsection{Site-direct mutagenesis}

A catalytic mutant of KLKB1 (S578A) was generated by site-direct mutagenesis. Overlap extension PCR was performed for generation of pCS4-3Flag-ITI-H4 $(\triangle \mathrm{BKD})$. The following primer sequences were used for generating mutants: KLKB1 (S578A); forward, 5'-TGT AAG GGA GAT GCA GGT GGT C-3', reverse, 5'-ACT AAG GGA CCA CCT GCA TCT C-3', BKD; forward, 5'-TGG CTC CCG GAT GAA TTT CAG ACG TCT GGC CAT CTT GCC TGC TT, reverse, 5'-AAG CAG GCA AGA TGG CCA GAC GTC TGA AAT TCA TCC GGG AGC CA-3'. After PCR reaction and gel purification, Dpn I enzyme (Enzynomics, Daejeon, Korea) was added and incubated for $1 \mathrm{~h}$. Then, PCR products were transformed into DH5 $\alpha$ competent cells, and DNA was extracted.

\subsection{RNAi interference}

siRNAs for ITI-H4 were generated with following sequences: \#1; 5'CGA ACC ACC CAU UUG AGA U-3', \#2; AUC UCA AAU GGG UGG UUC G, and KLKB1 siRNA; \#1; 5'-GUG CUU GCC AUC GAG ACA U-3', \#2; AUG UCU CGA UGG CAA GCA C-3' (Bioneer, Daejeon, Korea). 50 nM of ITIH4 siRNA and KLKB1 siRNA were introduced into the cells by transfection using Opti-MEM and RNAimax (Invitrogen, Paisley, UK) according to the manufacturer's instructions. The same concentration of nontargeting siRNA (Santa Cruz Biotechnology, sc-37,007) was used as a control.

\subsection{Cell counting kit-8 assay}

$100 \mu \mathrm{L}$ of transfected THP1 cell suspension having 50,000-100,000 cells/mL was added to 96 -well plate. The protocol was followed according to the instructions provided by the company (Dojindo Molecular Technologies, Rockville, MD, USA). O.D. was measured at $450 \mathrm{~nm}$ to determine the cell viability in each well.

\subsection{Transwell invasion and migration assay in vitro}

Invasion assays were performed in triplicate using transwell invasion chambers coated with Matrigel ( $50 \mu \mathrm{L}$ per filter) (BD Biosciences, Franklin Lakes, NJ, USA) as described in the manufacturer's protocol. Migration assays were performed using a 12-well Transwell chamber system (Costar 3422, Corning Inc., Oneonta, NY, USA). Cells were seeded in the upper chamber at $1.5 \times 10^{5}$ cells/mL in $0.1 \mathrm{~mL}$ serum-free RPMI- 1640 or DMEM media. After incubation for $24 \mathrm{~h}$ at $37{ }^{\circ} \mathrm{C}$ in an atmosphere containing $5 \% \mathrm{CO}_{2}$, migrated or invasive cells on the lower surface were stained with crystal violet stain and counted under a light microscope. All experiments were repeated six times over several days.

\subsection{Glutathione S-transferase (GST) pull-down assay}

ITI-H4 subcloned into glutathione S-transferase (GST)-tagged vector was transformed and expressed in BL21 E. coli strain. Bacterial lysate expressing GST-ITI-H4 was used to purify proteins using GlutathioneSepharose Beads (GE Healthcare, Buckinghamshire, England), according to the manufacturer's instructions. JEG-3 cells were transfected with Myc-KLKB1. After $24 \mathrm{~h}$ of incubation, the cells were harvested and 
suspended in Buffer A (20 mM Tris-HCl at pH 8.0, 1 mM EDTA, 1 mM dithiothreitol, $150 \mathrm{mM} \mathrm{NaCl}, 1 \%$ Triton X-100) containing $1 \times$ protease inhibitor mixture (Complete ${ }^{\mathrm{TM}}$ Roche Applied Science, Mannheim, Germany). Purified GST-ITI-H4 proteins were confirmed by Coomassie Brilliant Blue staining. To check the interaction between these proteins, GST or GST-ITI-H4 combined beads were incubated with JEG-3 cell lysates overexpressing Myc-KLKB1 and were tested by immunoblotting using anti-GST and anti-Myc antibodies.

\subsection{Data processing and statistical analysis}

Statistical comparison between patients with RPL and the controls was made using Student's t-test and Wilcoxon's test (SPSS 19.0). Pearson's or Spearman's correlation coefficient was calculated to estimate the interrelationships between gene expression levels and the distributions of relevant variables. $P$-values $<.005$ or $<.05$ were considered statistically significant. Comparisons between two groups of cells were made with a $t$-test (GraphPad Prism 4.0, GraphPad Software, Inc., San Diego, CA, USA). All experiments were repeated at least three times, and differences with $P$-values of $<.05$ were considered statistically significant.

\section{Results}

\subsection{ITI-H4 $\left(\Delta N^{688}\right)$ expression is elevated in sera of RPL patients}

To determine the change in ITI-H4 expression in the sera of RPL patients, we extended the number of samples with an additional set of 60 RPL patients and 30 controls. Interestingly, the long isoform of ITI-H4 expression was only found in normal control women. In contrast, the ITI-H4 $\left(\Delta \mathrm{N}^{688}\right)$ was present in approximately $50 \%$ of RPL patients (Supplementary Fig. S1a). We further measured serum levels of inflammatory markers (TNF- $\alpha$, IL-6, IL-4, IL-10, IL-13, IL-1 $\beta$, IFN- $\gamma$ and IL-2) with freshly collected blood samples of control subjects $(n=30)$ and patients with RPL $(n=60)$. Interestingly, the expression of proinflammatory cytokines/chemokines was significantly increased and the anti-inflammatory cytokine/chemokine was decreased in freshly collected blood samples of RPL patients compared to those of normal subjects (Supplementary Fig. S1b).

\subsection{The differential expression of ITI-H4 and KLKB1 in PBMCs of controls and RPL patients}

To verify the change in the level of ITI-H4 and KLKB1 in the PBMCs of RPL patients, we isolated PBMCs from 10 RPL patients and 10 controls. ITI-H4 mRNA levels were decreased in the PBMCs of RPL compared with those to the control group, while the mRNA levels of KLKB1 were significantly increased (approximately two-fold) in the PBMCs from RPL (Fig. 1A and Supplementary Fig. S2). In addition, the protein level of long isoform of ITI-H4 was reduced in RPL PBMC samples compared with control samples, whereas the protein level of ITI-H4 $\left(\Delta \mathrm{N}^{688}\right)$ in RPL PBMC samples was higher than in control samples (Supplementary Fig. S3a-d). Moreover, on the protein level, the expression of KLKB1 was significantly higher in RPL PBMC samples compared with control samples (Supplementary Fig. S4a-b). The imbalance of pro- and antiinflammatory cytokine expression might lead to recurrent miscarriage during pregnancy period, and previous reports have shown elevated concentrations of specific circulating pro-inflammatory markers in RPL patients [10]. We evaluated the status of inflammation in the PBMCs in the subjects by analyzing the mRNA levels of TNF- $\alpha$, IL-6, IL-4, IL-10, $I L-13, I L-1 \beta, I F N-\gamma$ and IL-2 in their PBMCs. As expected, the expression of pro-inflammatory genes at the mRNA level was clearly elevated in the PBMCs in the RPL group compared with that in the control group (Fig. 1B and Supplementary Fig. S5). Through a validated activity assay, we were able to confirm significantly increased level of KLKB1 in RPL patients (Fig. 1C). In addition, we also checked the correlation between ITI-H4 levels and inflammation status, and found that the ITI-H4
mRNA levels in PBMCs positively correlated with the expression of inflammatory factors (Supplementary Fig. S6).

\subsection{The enhanced expression of ITI-H4 $\left(\Delta N^{688}\right)$ and activation of IL-6 cas- cade modulators in human monocytes}

In the current study, we showed that IL-6 as an inflammatory mediated factor might lead to increased levels of ITI-H4 $\left(\Delta \mathrm{N}^{688}\right)$ and KLKB1 in human monocytic THP1 cells (Fig. 2A-2D). In addition, stimulation with IL-6 activates STAT3, ERK, and JNK within 30-60 min (Fig. 3A3D). The IL-6-mediated induction of ITI-H4 expression could be significantly decreased by treatment with an ERK inhibitor (PD98059) or a specific JNK inhibitor (SP600125), and a STAT3 inhibitor (LLL12) (Fig. 3E and F). Thus, these findings suggest that expression of ITI-H4 $\left(\Delta \mathrm{N}^{688}\right)$ is induced in human monocytic THP1 cells upon IL-6 stimulation in a STAT/MAPK-dependent manner.

Then, we determined whether inflammatory stimuli enhance the expression of ITI-H4 in human CD14+ monocyte cells, human CD14+ monocytes were treated with $20 \mathrm{ng} / \mathrm{mL}$ of IL-6 for $12 \mathrm{~h}$. Interestingly, IL-6 induces the activation of STAT3, ERK, and JNK pathway within 20-30 min (Supplementary Fig. S7a). Because ITI-H4 expression is induced by inflammation, we investigated the role of ITI-H4 in the inflammatory response. We examined the effect of ITI-H4 knockdown on the expression of inflammatory factors in human monocytes (Supplementary Fig. S7b and S8). The expression of these pro-inflammatory genes (TNF- $\alpha, I L-6, I L-1 \beta$, and IFN- $\gamma$ ) were all markedly induced and the antiinflammatory genes (IL-4, IL-10, IL-13, and IL-2) were downregulated after knockdown of ITI-H4. These results suggest that ITI-H4 expression is enhanced in human CD14+ monocytes by treatment of inflammatory stimuli in an IL-6-dependent manner. Furthermore, ITI-H4 could positively or negatively regulate the Th1 and Th2 cytokines with potential pro- and anti-inflammatory activities. A previous report suggests that pig MAP is an IL-6-dependent acute-phase protein in porcine primary cultured hepatocytes [17]. Thus, we hypothesized that the human ITI$\mathrm{H} 4$ is also regulated by IL- 6 in human hepatocellular cells. To confirm this effect in a quantitative manner, we treated hIL- 6 at doses of 100 and $1000 \mathrm{U} / \mathrm{mL}$ for various time intervals $(0,2,6,24$, and $48 \mathrm{~h})$ in human hepatocellular cells. Interestingly, we found that IL- 6 induced an increase of human ITI-H4 both at the MRNA and protein levels. However, we could not detect the ITI-H4 $\left(\Delta \mathrm{N}^{688}\right)$ in human hepatocellular cells (Supplementary Fig. S9a-d).

To investigate cellular localization of ITI-H4, we performed immunofluorescent staining of endogenous ITI-H4. Our result revealed that IL-6 altered ITI-H4 expression and translocation. Thus, we further checked ITI-H4 localization in the presence of IL-6. Without IL-6 treatment, ITIH4 is mainly localized in the nucleus (Supplementary Fig. S10a, middle panel). However, it is of interest that the nuclear localization of ITI-H4 is abrogated in IL-6-induced condition (Supplementary Fig. S10b, middle panel, 10 ng/mL; Supplementary Fig. S10c, middle panel, 100 ng/mL.

\subsection{ITI-H4 interacts with KLKB1, and KLKB1 stabilizes ITI-H4 protein in a dose dependent manner}

The C-terminus of human ITI-H4 contains a proline-rich region (PRR; Gly ${ }^{611}-\mathrm{Gln}^{730}$ ), which extends 120 residues in length and harbors species- and tissue-specific domains [19,20]. In addition, protein von Willebrand factor type A (Vwf) domains are typically involved in protein-protein interactions and glycosylation at this site might potentially affect ITI-H4 interactions with other proteins [21]. Moreover, the bradykinin-like fragment ( Pro $^{622}-\mathrm{Arg}^{688}$ ) within the PRR may play an important role in inflammatory response during acute inflammation [22]. The detailed domain information of ITI-H4 is illustrated (Supplementary Fig. S11a). Bradykinin domain (BKD) is the only domain located towards the C-terminal region of ITI-H4. Interestingly, the BKD domain-deleted ITI-H4 showed relatively less interaction with KLKB1 compared to the long isoform of ITI-H4 (Supplementary Fig. S11b). In 
A

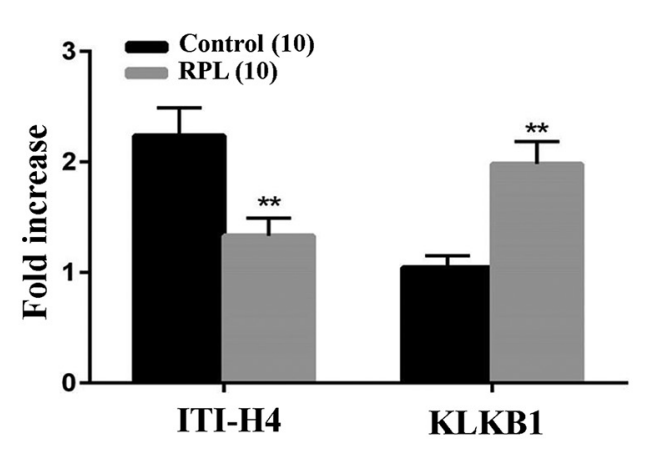

B

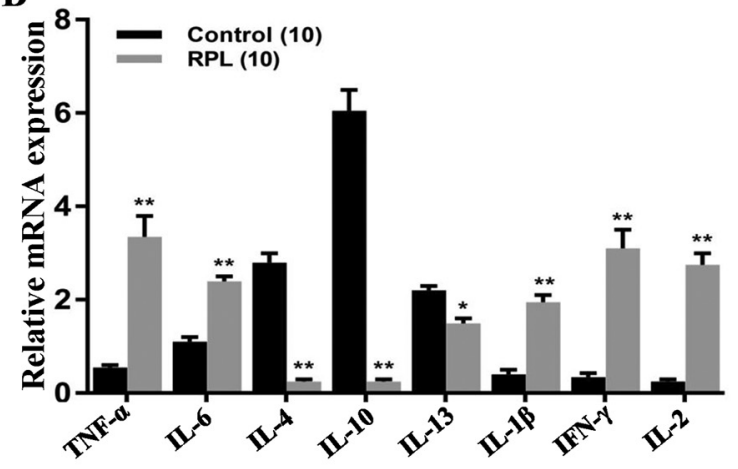

C

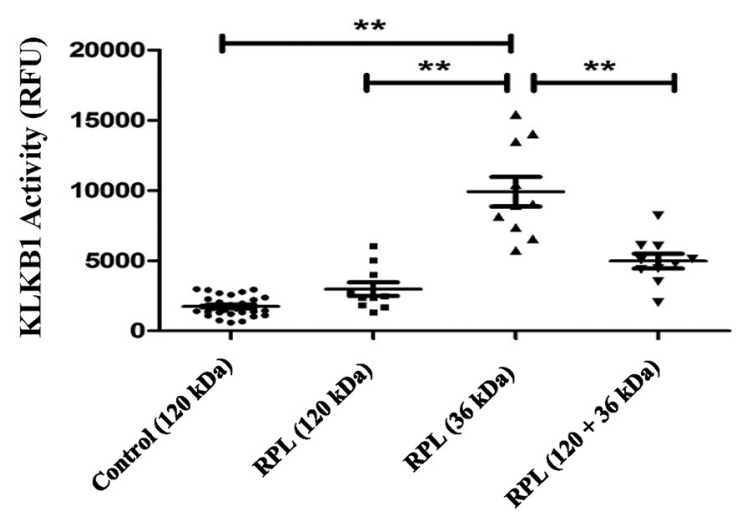

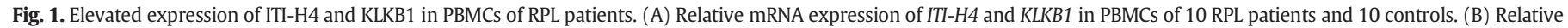

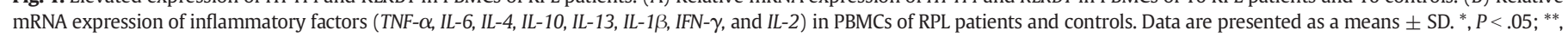

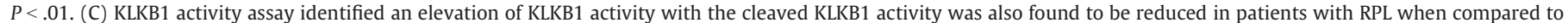
controls. Data are presented as a means $\pm \mathrm{SD}$. ${ }^{*}, P<.05$; $^{* *}, P<.01$.

A

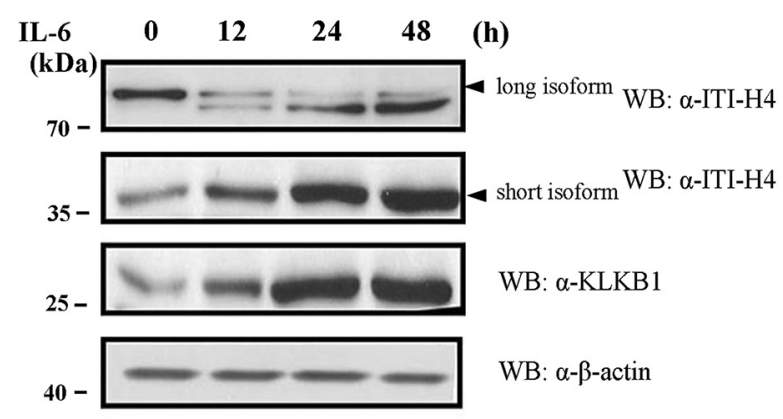

C

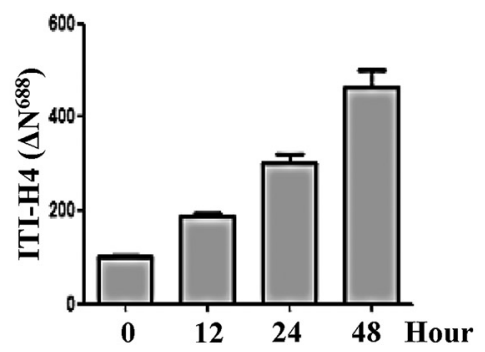

B

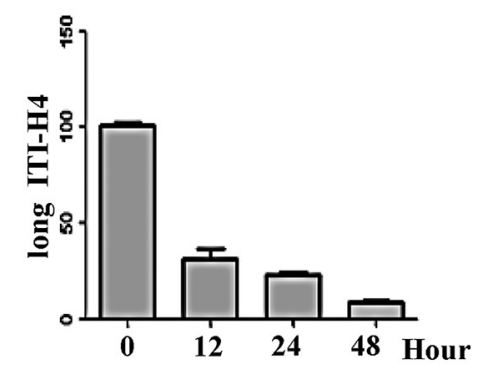

D

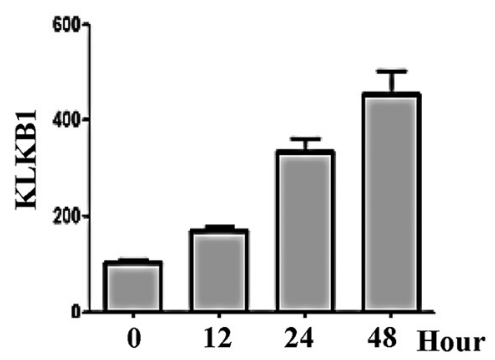

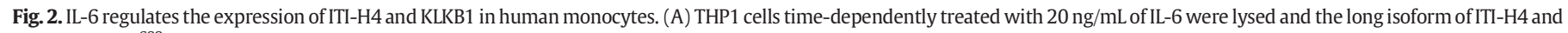

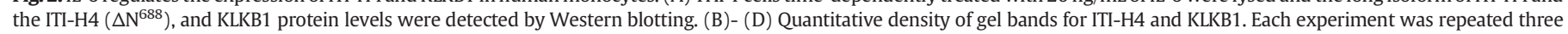
times. 
A

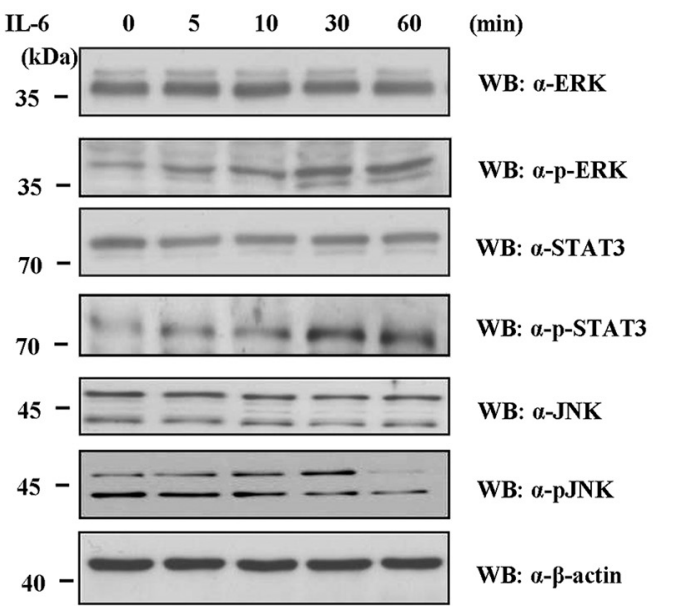

B

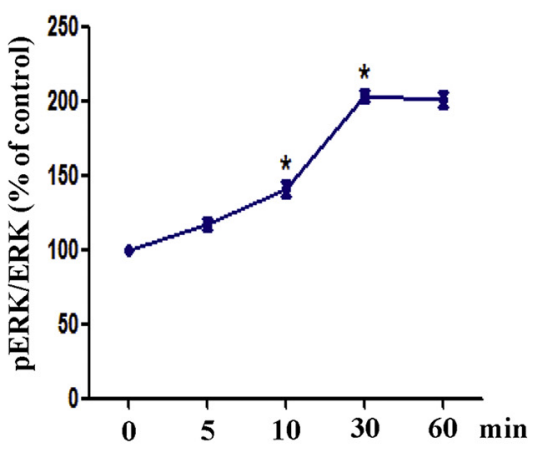

$\mathbf{E}$

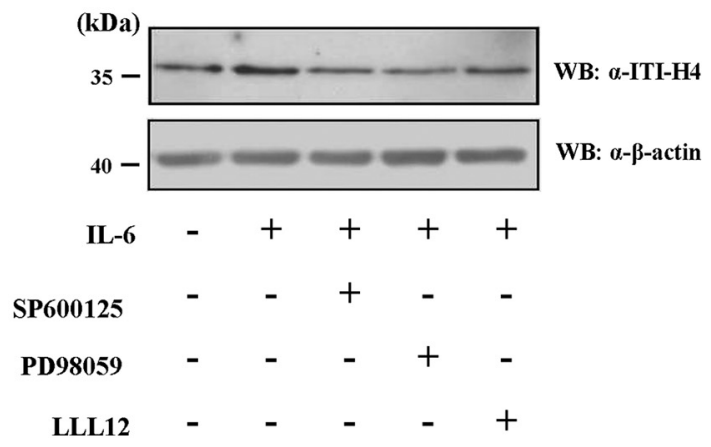

C

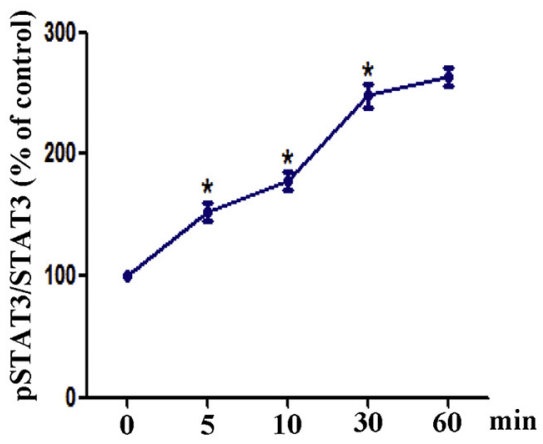

$\mathbf{F}$

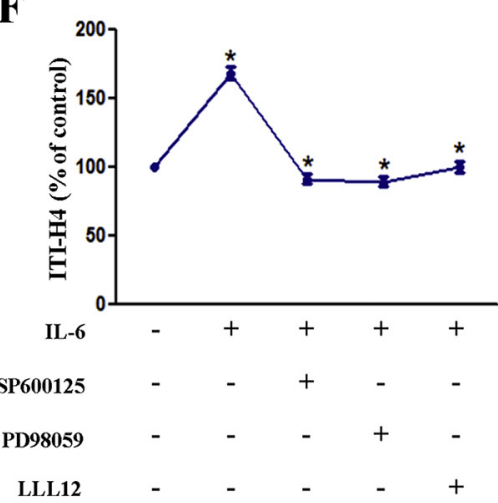

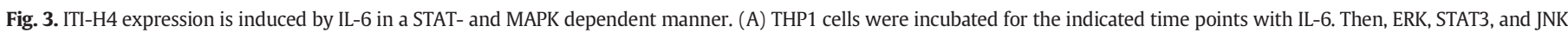

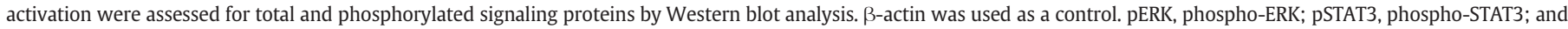

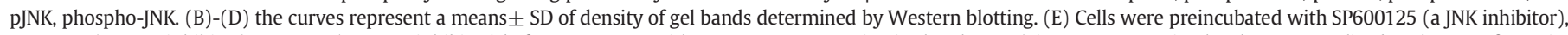

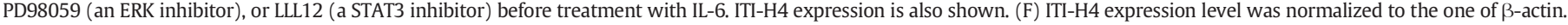
All statistical data are presented as a means $\left(n=3,{ }^{*}, P<.05\right)$.

addition, we found that an anti-ITI-H4 antibody could co-precipitate endogenous KLKB1, and the level of ITI-H4 protein dramatically decreased in the presence of KLKB1 in a dose dependent manner (Supplementary Fig. S11c-11d). However, a catalytic mutant KLKB1 (S578A) failed to reduce the level of ITI-H4 (Supplementary Fig. S11e). In a GST pull-down assay, KLKB1 from cell lysates was pulled down by GST-ITI-H4 bound beads, suggesting that ITI-H4 directly binds to KLKB1 (Supplementary Fig. S11f). Our result suggests that the KLKB1 acts as a specific protease for ITI-H4 regulating its protein stability.

\subsection{ITI-H4 regulates pro- and anti-inflammatory cytokines/chemokines in} human placental choriocarcinoma cells

To investigate the role of ITI-H4 in the inflammatory response in human choriocarcinoma cells, we examined the effect of ITI-H4 knockdown on the expression of inflammatory factors in JEG-3 cells (Fig. 4A, B and Supplementary Fig. S12). The expression of these pro-inflammation-related genes (TNF- $\alpha$, IL-2, IL-1 $\beta$, and IL-6) were markedly induced, while anti-inflammation-related genes (IL-4, $I L-10$, and IL-13) were clearly inhibited after down-regulation of ITI-H4, indicating that ITI-H4 is involved in modulating inflammation in human placental choriocarcinoma cells. These results show that ITI-H4 regulates the pro- and anti-inflammatory genes in human choriocarcinoma cells.
3.6. The opposing inflammatory functions of two different isoforms of ITIH4

To further investigate the molecular inflammatory roles of the long isoform of ITI-H4 and ITI-H4 $\left(\Delta \mathrm{N}^{688}\right)$, we examined the effect of overexpression of two different forms in human placental choriocarcinoma cells. The expression of these pro-inflammatory-related cytokines (TNF- $\alpha$, IFN- $\gamma$, IL-1 $\beta$, IL-2, and IL-6) was significantly increased after the cells were transfected with the ITI-H4 $\left(\Delta \mathrm{N}^{688}\right)$. Nevertheless, antiinflammatory cytokines (IL-4, IL-10, and IL-13) were upregulated after the cells were transfected with the long isoform of ITI-H4 (Fig. 5A-5C). Additionally, our data suggest that ITI-H4 might be regulated by KLKB1 in vitro and in vivo. We also checked the inflammatory roles of KLKB1 and its catalytic mutant KLKB1 (S578A) (Fig. 5D). Interestingly, KLKB1 overexpression enhanced the production of pro-inflammatory cytokines/chemokines which might be involved in inflammatory response. Furthermore, overexpression of the long isoform of ITI-H4 and KLKB1 resulted in significant increase of pro-inflammatory cytokines expression, while transfection of the long isoform of ITI-H4 along with KLKB1 (S578A) caused upregulation of anti-inflammatory cytokines compared with the co-transfection of the long isoform of ITI-H4 and KLKB1 (Fig. 5E), indicating specific protease activity of KLKB1 on ITIH4. Consequently, our results are suggestive of KLKB1's ability to interact and stabilize ITI-H4 expression to affect immune response and production of cytokines/chemokines. The expression level of individual 
A

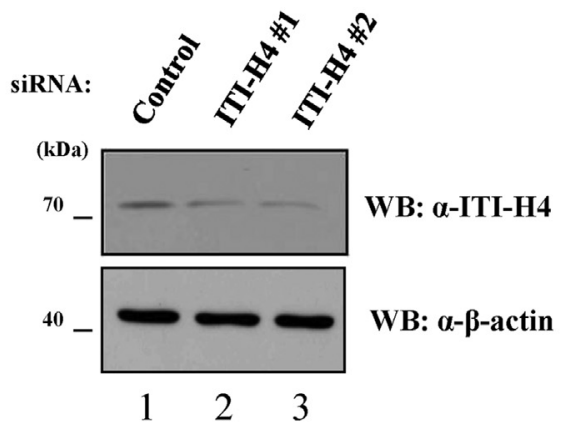

B

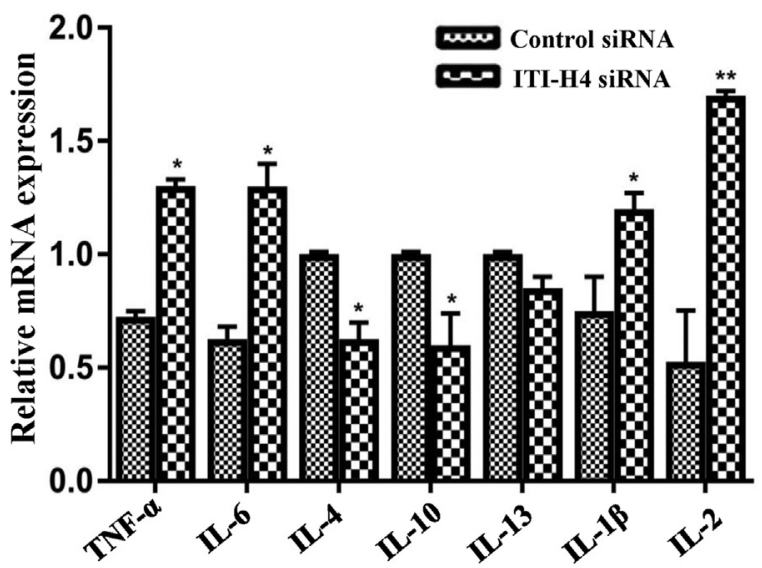

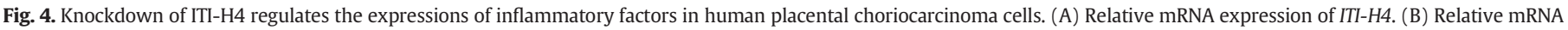
expression of inflammatory factors after ITI-H4 siRNA treatment in human placental choriocarcinoma cells. Data are presented as a means \pm SD $(\mathrm{n}=3) .{ }^{*}, P<.05$.

constructs was checked by Western blot analysis (Supplementary Fig. S13a-13c).

3.7. ITI-H4 $\left(\Delta N^{688}\right)$-induced cytokine and chemokine expression is dependent on STAT and MAPK pathways

Further to determine the signaling cascade, we examined the activation of STAT, ERK, and JNK molecules after overexpression of the ITI-H4 $\left(\Delta \mathrm{N}^{688}\right)$ by Western blotting (Fig. $\left.5 \mathrm{~F}\right)$. Phosphorylation of STAT3 and ERK was enhanced after transfection with ITI-H4 expression vector, while a slight change of phosphorylation of JNK was detected after ITI$\mathrm{H} 4\left(\Delta \mathrm{N}^{688}\right)$ overexpression (Fig. 5G and Supplementary Fig. S14). Remarkably, the ERK phosphorylation inhibitor PD98059, the JNK phosphorylation inhibitor SP600125, and STAT3 phosphorylation inhibitor LLL12 blocked the increase in the ITI-H4 $\left(\Delta \mathrm{N}^{688}\right)$, which reduced the activation of STAT, ERK, and JNK, compared to their normal activation levels (Fig. $5 \mathrm{H}$ ). Taken together, these results suggest that ITI-H4 $\left(\Delta \mathrm{N}^{688}\right)$-induced inflammation and immune response are dependent on the STAT, ERK, and JNK signaling pathways.

\subsection{ITI-H4 and KLKB1 regulate cell invasion in human placental choriocar- cinoma cells}

Several lines of evidence have suggested that IL-6/STAT3 pathway affects cell invasion and migration [23-25]. Analysis of invasion behavior of the transfected JEG-3 cells indicates that the ITI-H4 $\left(\Delta \mathrm{N}^{688}\right)$ - or KLKB1-transfected cells showed significant reduction in invasion when compared to that of the controls. The co-transfection of the long isoform of ITI-H4 and KLKB1 displayed significantly reduced invasion in comparison to that of cells transfected with the long isoform of ITI$\mathrm{H} 4$ alone (Fig. 6A and B). Western blotting was performed to determine the exogenous level of the long isoform of ITI-H4, ITI-H4 $\left(\Delta \mathrm{N}^{688}\right)$, and KLKB1 (Supplementary Fig. S15a). In addition, knockdown of both ITI$\mathrm{H} 4$ and KLKB1 expression resulted in an increase in the number of invasive cells compared with the ITI-H4 knockdown alone (Fig. 6C and D). The designed siRNAs for ITI-H4 and KLKB1 effectively depleted endogenous ITI-H4 and KLKB1 in JEG-3 cells (Supplementary Fig. S15b). Taken together, it is suggested that the ITI-H4 $\left(\Delta \mathrm{N}^{688}\right)$ could inhibit cell invasion, and the functional interaction between ITI-H4 and KLKB1 is essential for the regulation of cell invasion.
3.9. The ITI-H4 $\left(\Delta N^{688}\right)$-overexpressed JEG-3 cells could enhance macrophage migration

After confirming the induction of ITI-H4 expression by inflammatory response, we next questioned the role of the long isoform of ITI-H4 and the ITI-H4 $\left(\Delta \mathrm{N}^{688}\right)$ in the inflammatory response. We investigated the effect of ITI-H4 on macrophage migration, which is a hallmark of the immune response for disposal invasions. As mentioned in Materials and Methods, JEG-3 cells were transfected with Flag-tagged long ITI-H4 and ITI-H4 $\left(\Delta \mathrm{N}^{688}\right)$ along with the empty Flag vector, and were placed in the bottom wells for $36 \mathrm{~h}$. Macrophages were resuspended after overnight culture in serum-free RPMI-1640, and then added to the upper chamber in serum-free RPMI-1640. After $4 \mathrm{~h}$ of incubation, the number of migrating cells was determined. Interestingly, the long isoform of ITIH4-overexpressed JEG-3 cells could successfully inhibit chemotactic migration of human macrophage, while the ITI-H4 $\left(\Delta \mathrm{N}^{688}\right)$-overexpressed JEG-3 cells could enhance human macrophage migration (Fig. 6E and F), indicating that the ITI-H4 $\left(\Delta \mathrm{N}^{688}\right)$ acts as a chemoattractant to drive integration of cytokines and chemokines in human JEG-3 cells. Western blotting was performed to determine the exogenous level of the long isoform ITI-H4 and ITI-H4 ( $\left.\Delta \mathrm{N}^{688}\right)$ (Supplementary Fig. S15c).

\subsection{Cell proliferation assay}

Our data suggest that the ITI-H4 $\left(\Delta \mathrm{N}^{688}\right)$ could promote macrophage migration, which might modulate immune response. To further investigate the effect of the long isoform of ITI-H4 and ITI-H4 $\left(\Delta \mathrm{N}^{688}\right)$ on monocyte proliferation, we next conducted cell proliferation assay using cell counting kit- 8 over $72 \mathrm{~h}$ in three separate experiments. Interestingly, the long isoform of ITI-H4 showed significant inhibition on human monocyte (THP1) cell proliferation, and the KLKB1 regulation of the long isoform of ITI-H4-mediated THP1 cell proliferation was identified (Fig. 7A). The ITI-H4 $\left(\Delta \mathrm{N}^{688}\right)$ could promote THP1 cell proliferation in contrary to the long isoform of ITI-H4 (Fig. 7B). The expression level of individual constructs was checked by Western blot analysis (Supplementary Fig. S16a and 16b). Under the condition of ITI-H4 or KLKB1 gene silencing by siRNA, the block in cell proliferation caused by the long isoform of ITI-H4 greatly reduced (Fig. 7C). In addition, we observed possibility of KLKB1 regulation of THP1 cell proliferation (Fig. 7C). The knockdown efficiency of silTI-H4 and siKLKB1 was checked by Western blot analysis in THP1 cells (Supplementary Fig. S16c). Collectively, our data showed that the long isoform of ITI- 
A

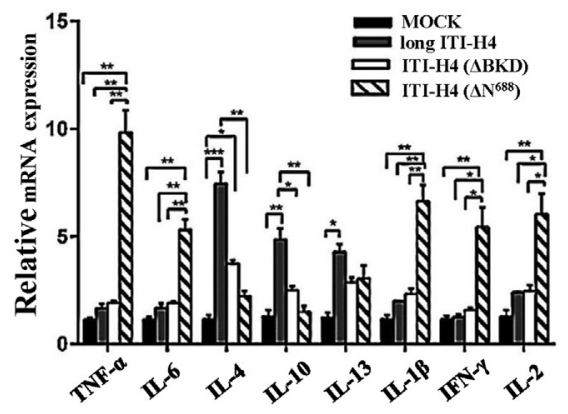

D

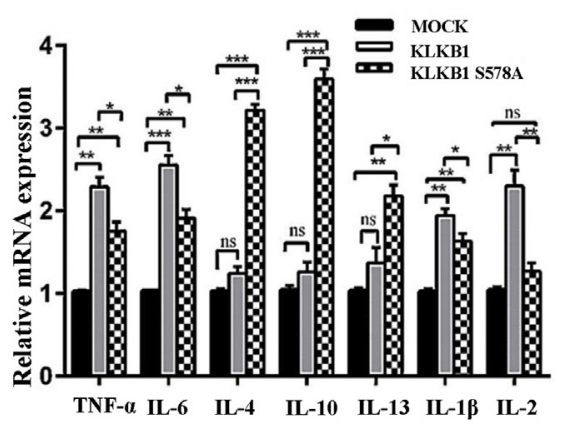

B

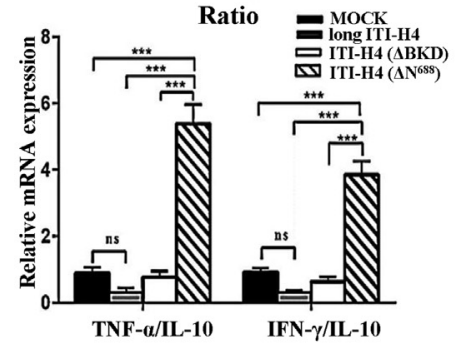

$\mathbf{E}$

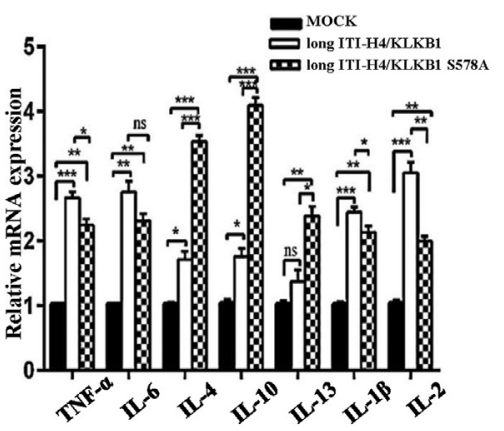

C
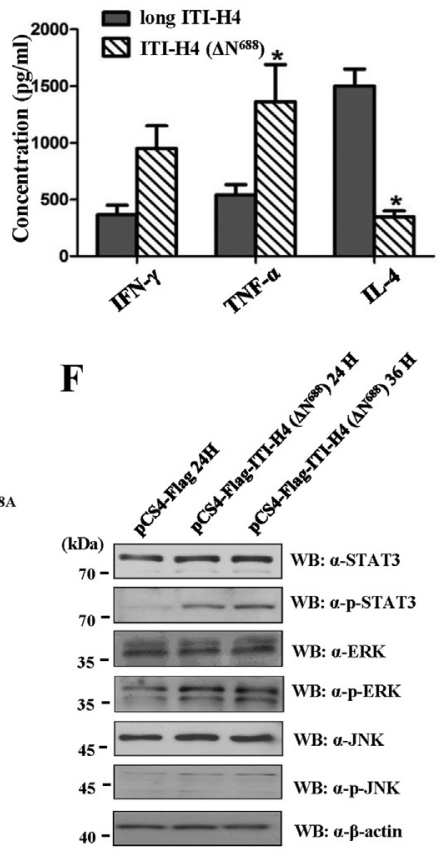
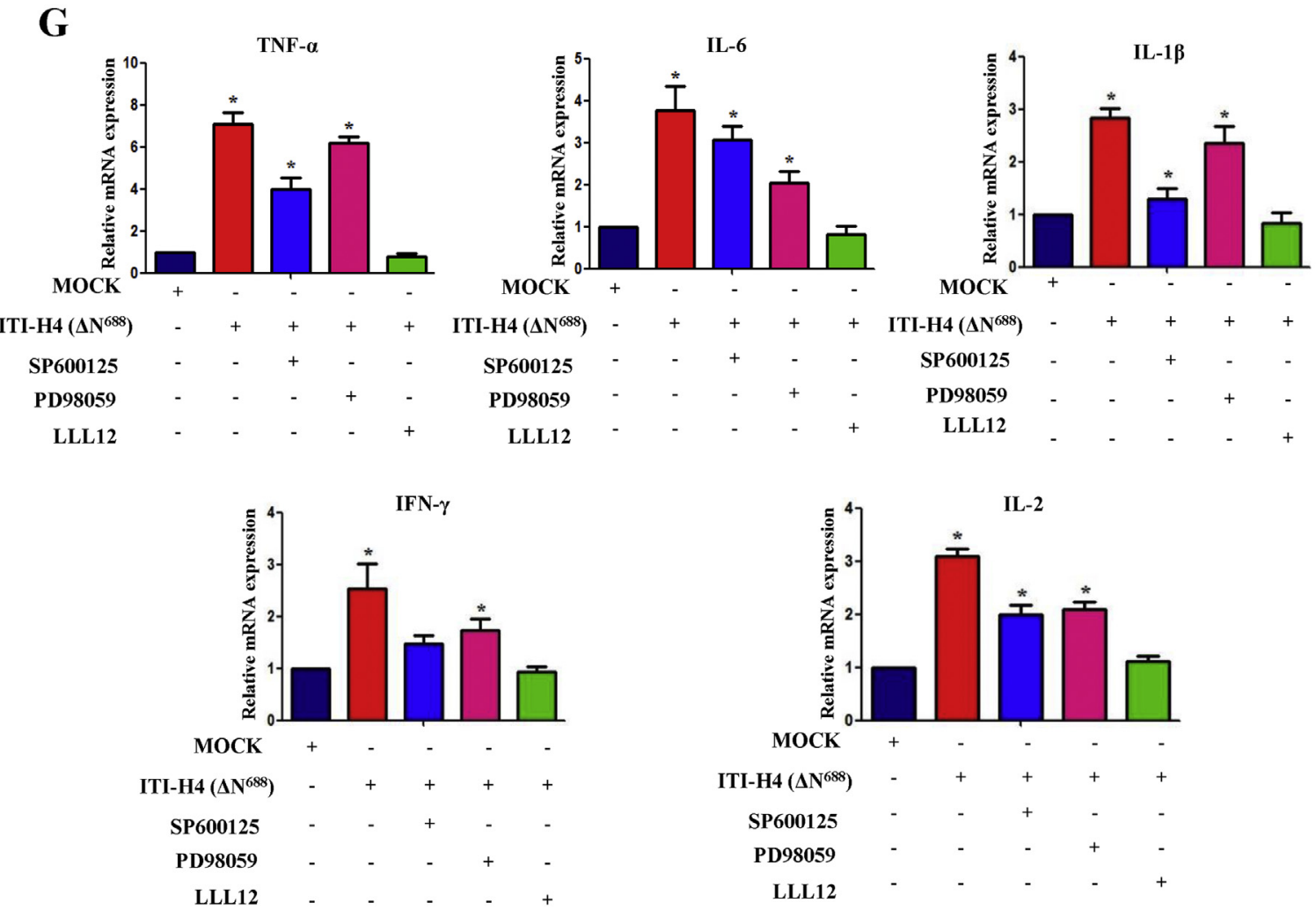

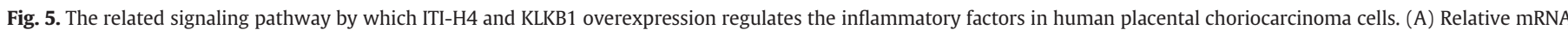

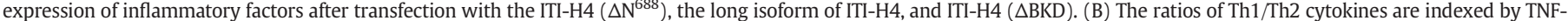

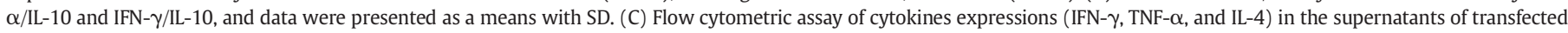

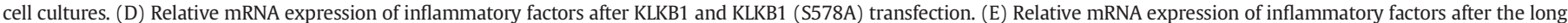

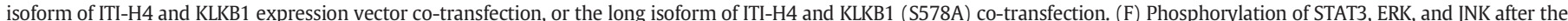

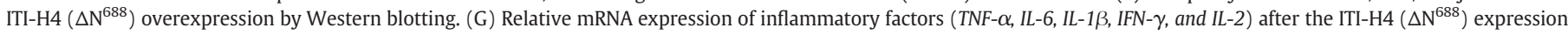

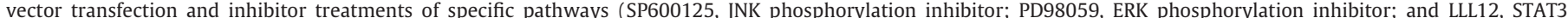

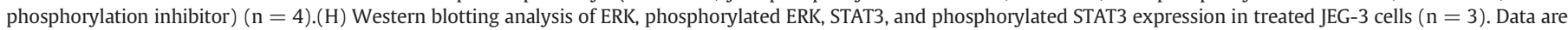

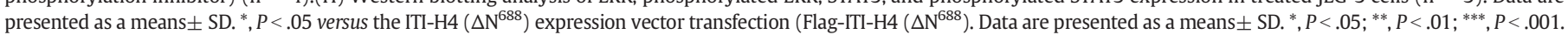


H

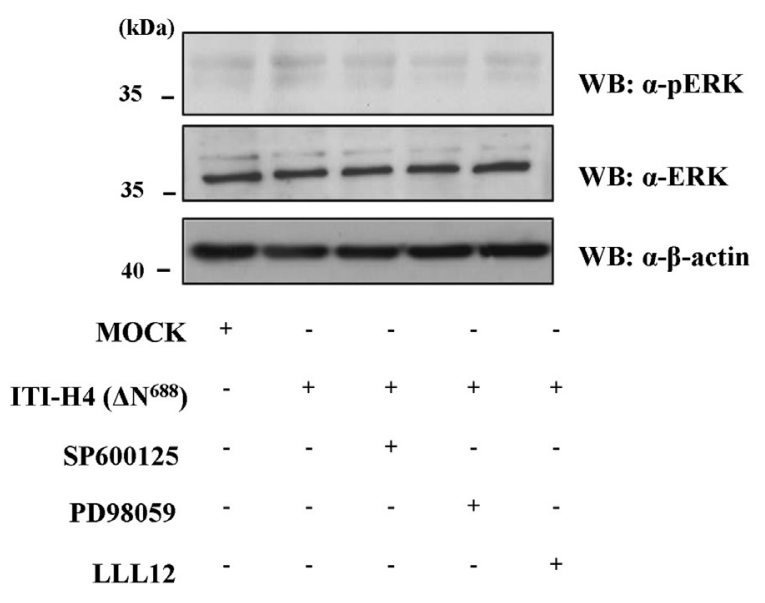

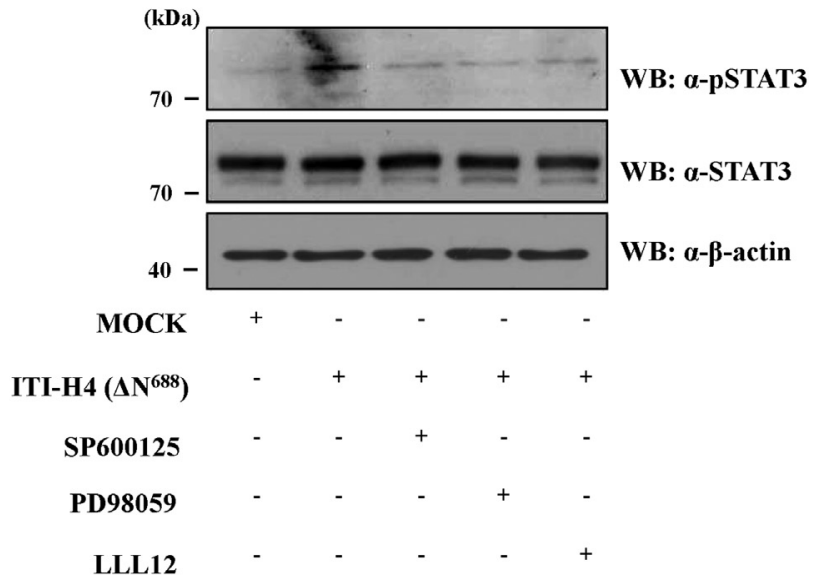

Fig. 5 (continued).

$\mathrm{H} 4$ and ITI-H4 $\left(\Delta \mathrm{N}^{688}\right)$ might have the opposing effects on human monocytic cell proliferation. Intriguingly, KLKB1 and ITI-H4 mutually interact with each other to regulate cell proliferation.

\subsection{Co-culture with human choriocarcinoma cells and serum derived from RPL patients or normal groups}

We hypothesized that the ITI-H4 $\left(\Delta \mathrm{N}^{688}\right)$ might be closely interconnected or involved in the pathogenesis of RPL, since the ITI-H4 $\left(\Delta \mathrm{N}^{688}\right)$ was highly expressed in the serum derived from RPL patients. For this purpose, we investigated the functional role of ITI-H4 using the coculturing system. JEG-3 cells were separately cultured in DMEM medium supplemented with $5 \% \mathrm{FBS}$, and serum (5\%) pooled from RPL patients or normal women groups for $24 \mathrm{~h}$. Each group contains 6-7 women. The long isoform of ITI-H4 was highly expressed in both normal group 1 and 2 , and the ITI-H4 $\left(\Delta \mathrm{N}^{688}\right)$ was highly expressed in all of patients belonging to the RPL group 1 and 2. Based on our previous study, we chose group 3 serum samples which showed both forms of ITI-H4. In addition, the captured microscopy images were shown in Fig. 7D. Strikingly, we observed 'jelly coat' type in normal women group 1 and 2 (Supplementary Fig. S17a and Video 1-4. Mp4), and approximately $70-80 \%$ of cell death was quantified by trypan blue staining in RPL group 1 and 2 . Then, we measured relative cytokine expressions in those samples. As expected, the pro-inflammatory cytokines (TNF- $\alpha$, $I L-2$, and $I F N-\gamma$ ) are increased in RPL group samples, while the antiinflammatory cytokines (IL-4 and IL-10) were upregulated in normal group samples (Fig. 7E). Altogether, to some extent, our findings demonstrate that ITI-H4, particularly the ITI-H4 $\left(\Delta \mathrm{N}^{688}\right)$ that was present in the RPL serum, could upregulate pro-inflammatory cytokines. Additionally, invasion analysis indicates that the RPL group 1 and 2cocultured cells showed significant reduction in invasion when compared to that of the normal groups 1 and 2 (Supplementary Fig. S17b and S17c). Similarly, high expression of matrix metalloproteinase-9 (MMP-9), but not MMP-2, mucin 1 (MUC1) or human placental protein (PP14), was observed in RPL groups 1 and 2 (Supplementary Fig. S17d and S18).

\section{Discussion}

There are increasing lines of evidence suggesting a role of chronic or acute inflammation in the pathogenesis of RPL, which is demonstrated by persistent, moderately elevated concentrations of cytokines, chemokines and circulating C-reactive protein. The paradoxical success of the "fetal allograft" in the face of a potentially hostile maternal immune system has been suggested to be due to immunomodulation at the maternal-fetal interface and a consequent lack of strong maternal cell-mediated anti-fetal reactivity of the Thelper 1 (Th1) type [26,27]. Several studies on animal models of pregnancy and a few on human pregnancies have led to the proposition that successful pregnancy is a Thelper 2 (Th2) phenomenon, while vigorous Th1-type immunity, mediated by Th1-type cytokines, is deleterious to pregnancy $[28,29]$. Therefore, the balance of Th1/Th2 cytokines is essential to maintain pregnancy. Here, we observed the high expression of TNF- $\alpha$, IL-2, IL$1 \beta$, and IFN- $\gamma$ which belong to Th1 cytokines in RPL patients, while Th2 cytokines, particularly like IL-4 and IL-10, are upregulated in control group. In addition, we observed the long isoform of ITI-H4 in normal women; however, approximately $76.9 \%$ of ITI-H4 $\left(\Delta \mathrm{N}^{688}\right)$ was detected in RPL women [12]. Compared to our previous study [12], the current study enlarges the sample size to 30 controls and 60 RPL patients; nevertheless, the ITI-H4 $\left(\Delta \mathrm{N}^{688}\right)$ still occupies a large proportion in RPL patients. Moreover, increased the expression of KLKB1 and decreased mRNA expression of ITI-H4 in PBMCs of RPL patients compared with those in the controls, and the ITI-H4 expression patterns were correlated with the expression of inflammatory markers. It is reasonable to surmise that an imbalance between ITI-H4 and KLKB1 levels is associated with inflammation in RPL patients. The role of ITI-H4 in RPL remains undetermined and no functional mechanism study has yet been performed that concerns RPL. The structure of the proteins of ITI family has been widely studied [30,31]. Because of its sensitivity to kallikrein degradation, it was later considered as a kallikrein substrate, and known as PK-120 [32]. ITI-H4 has several putative domains, and previous studies have demonstrated that some of these domains were also found to be associated with different diseases [33]. In addition, the bradykinin-like fragment ( $\mathrm{Pro}^{662}-\mathrm{Arg}^{688}$ ) within the PRR is assumed to take part in inflammatory response during acute inflammation [23]. The BKD contains the KLKB1 cleavage site (R688). In our previous LCMS/MS analysis and the current study have identified the cleavage site of ITI-H4 fragmentation [12]. Further domain functional studies also suggested that the BKD domain might play important roles in immune response which might be involved in the pathogenesis of RPL.

Furthermore, it is worth noting that elevated IL-6 is frequently evident in the altered cytokine profiles characteristic of unexplained infertility, recurrent miscarriage, and preterm delivery [28]. In addition, RPL is accompanied by increased IL-6 trans-signaling systemically, yet it is still controversial [34]. IL-6 is a multifunctional cytokine with pivotal roles in the inflammatory response and there is significant relationship between IL-6 signaling components and $\mathrm{T}$ cell responses in pregnant women [34]. And excessive IL-6 bioavailability potentially inhibiting generation of $\mathrm{CD} 4+\mathrm{T}$ regulatory cells is required for pregnancy tolerance [28]. Interestingly, IL-6 as an inflammatory mediated cytokine could 
A

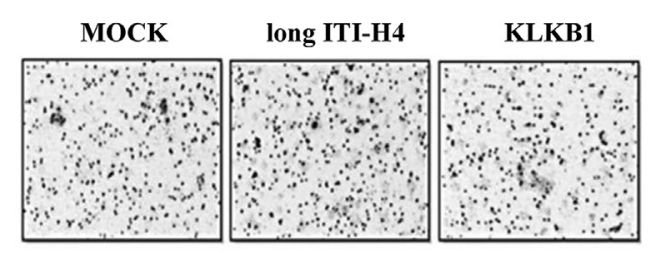

ITI-H4 $\left(\Delta \mathrm{N}^{688}\right)$ long ITI-H4/KLKB1
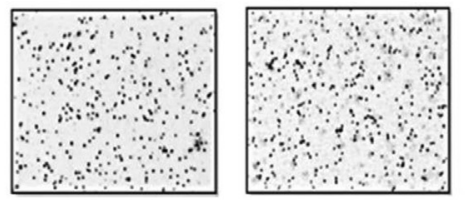

D

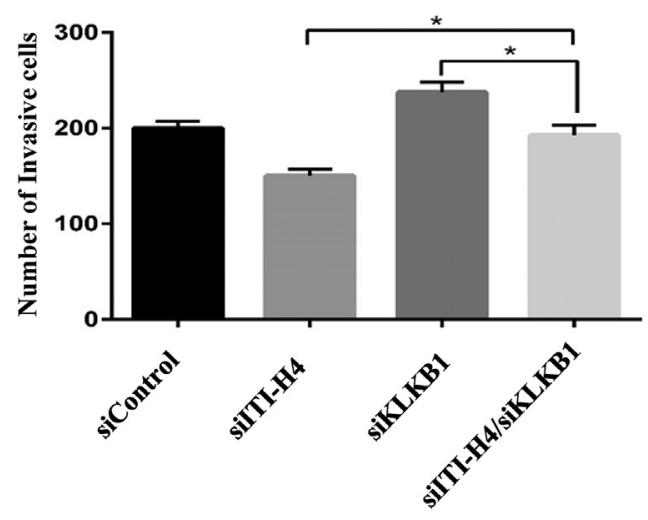

B

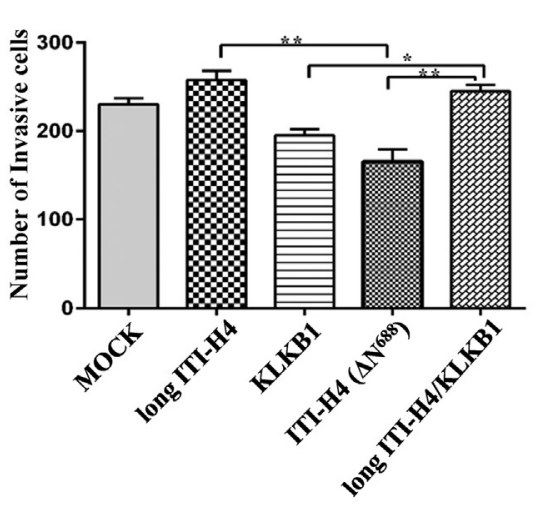

C

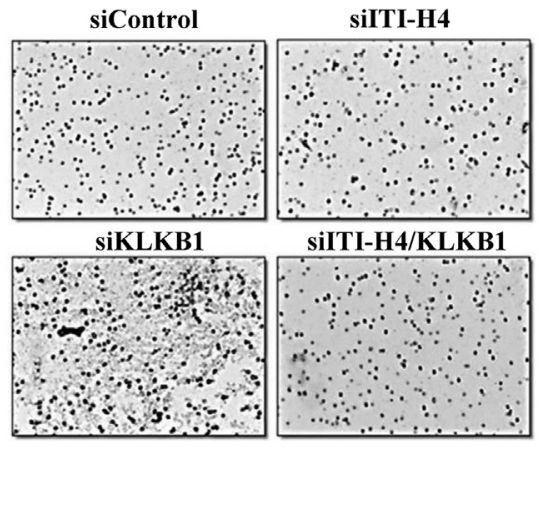

$\mathbf{E}$
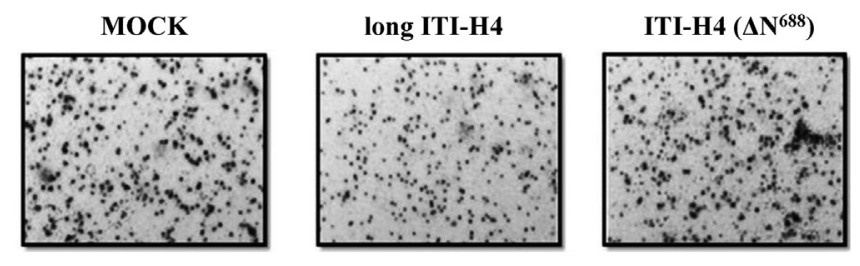

$\mathbf{F}$

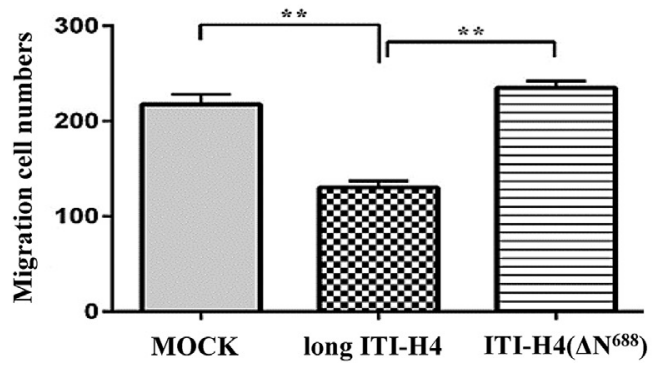

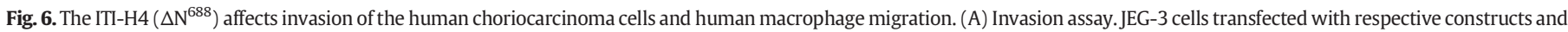

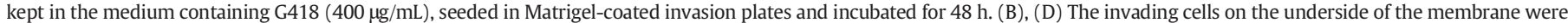

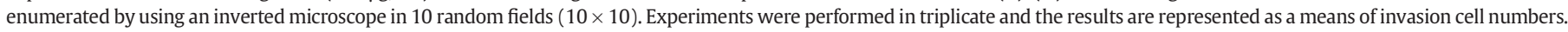

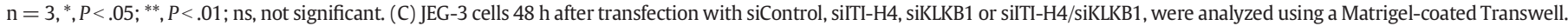

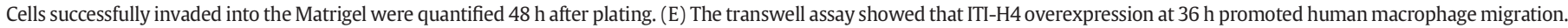
(F) Data are presented as a means $\pm \mathrm{SD}(\mathrm{N}=3) .{ }^{*}, P<.05,{ }^{* *}, P<.01$, versus control vector transfection (Flag group).

increase the KLKB1 level and stimulate the production of ITI-H4 $\left(\Delta \mathrm{N}^{688}\right)$, while the long isoform of ITI-H4 was decreased in monocytes. We also observed IL-6 activates the downstream signaling factors of STAT and MAPK pathways in human CD14+ monocytes. In addition, knockdown of ITIH4 in human CD14+ monocytes could upregulate pro-inflammatory cytokines expression. Additionally, challenge with the inflammatory cytokine IL-6 could upregulate the expression of ITI-H4 $\left(\Delta \mathrm{N}^{688}\right)$, and SP600125, PD98059, or LLL12 inhibitors blocked this response.

Our further functional studies have also demonstrated that expression of Th1 cytokines was increased after overexpression of the ITI-H4 $\left(\Delta \mathrm{N}^{688}\right)$, while Th2 cytokines were upregulated after the cells were transfected with the long isoform of ITI-H4. Our data suggest that the long isoform of ITI-H4 might play positive roles in the maintenance of Th1/Th2 balance, while ITI-H4 $\left(\Delta \mathrm{N}^{688}\right)$ does not (Fig. $\left.7 \mathrm{~F}\right)$.

In addition, IL-6 plays a regulatory role in trophoblast invasion, migration or proliferation [35]. It has been suggested that ITI-H4 may have a role in protecting the uterus from the inflammatory response induced by conceptus attachment to the uterine epithelium [35]. To gain a better insight into the mechanism underlying ITI-H4 involved in the pathogenesis of RPL, we hoped to identify the functional differences between the long isoform of ITI-H4 and ITI-H4 $\left(\Delta \mathrm{N}^{688}\right)$ regarding immune modulation, trophoblast cell invasion, immune cell migration or proliferation.

Moreover, additional invasion analysis using above co-culturing system, the RPL groups-cocultured cells showed decreasing invasion when compared to that of the normal groups. And we also observed the expression of immunity-related genes was decreased in the RPL groupcocultured cells, such as PP14 and MUC1. In our previous studies have also suggested that these factors were aberrantly expressed in chorionic villi from RPL patients [10]. Approximately 25\% of RPL patients has shown the elevation of immune response to trophoblast and increased proliferation of inflammatory cells [36]. This leads to the possibility that aberrant regulation of immunosuppression-related gene products may cause RPL. PP14 has important roles as a possible immunosuppressive molecule capable of successful pregnancy and growth of the fetus [3]. In addition, adhesion molecules such as MUC1 play an important role in apposition and adhesion, followed by trophoblast invasion through the pinopode formation [37]. It has also been suggested that decreased expression of MUC1 might induce endometrial superfertility and interrupt embryo selection, which allows defective blastocysts to implant but leads to increase miscarriage rate [38]. Moreover, MMP-2 and MMP-9 were found to be involved in successful cytotrophoblast invasion in early pregnancy [39]. We suggest that 'jelly-coat' phenotype formation in normal women groups might be closely related to the normal immune response or invasion, while the RPL group-cocultured might be involved in a strong inflammatory response in pregnant women, and consequently the pregnancy may fail.

Taken together, it is suggested that the long isoform of ITI-H4, ITI-H4 $\left(\Delta \mathrm{N}^{688}\right)$, and KLKB1 play important roles in the maintenance of 
A

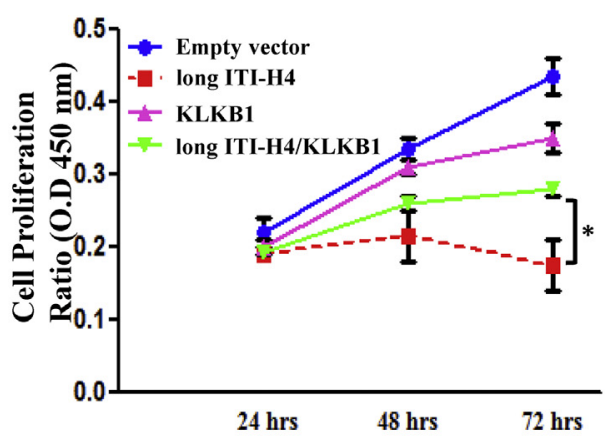

D

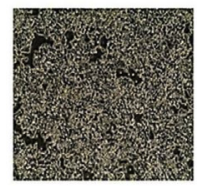

Control FBS 5\%

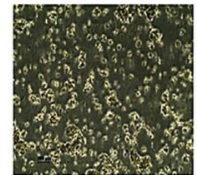

RPL Group 1

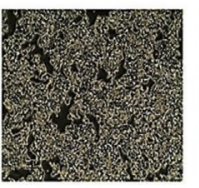

Normal Group 1

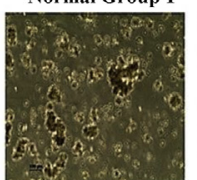

RPL Group 2

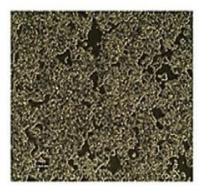

Normal Group 2

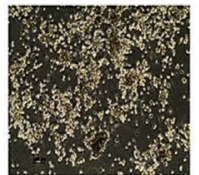

RPL Group 3
B

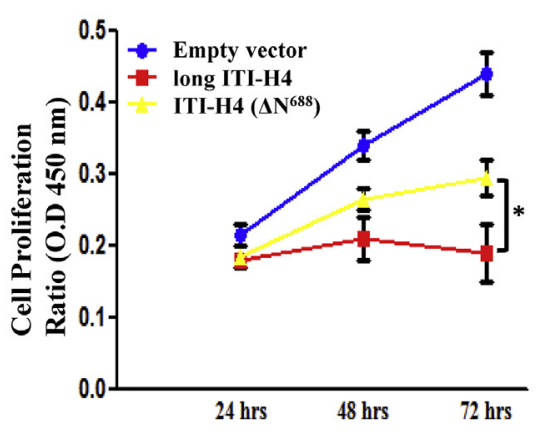

E

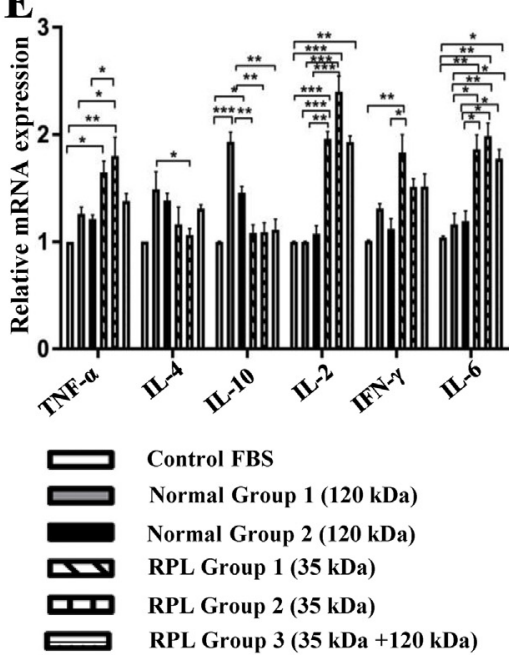

C

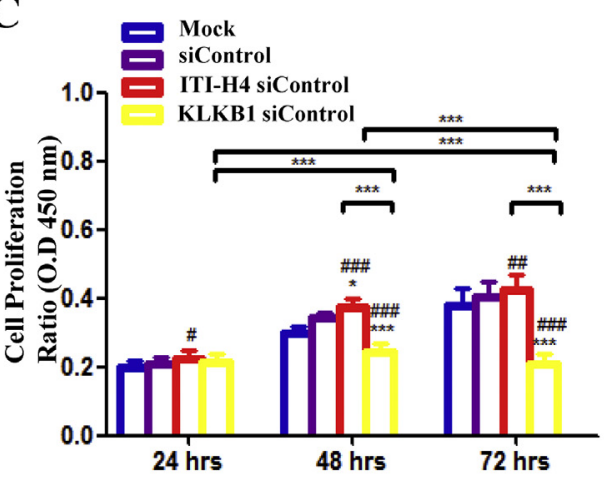

$\mathbf{F}$

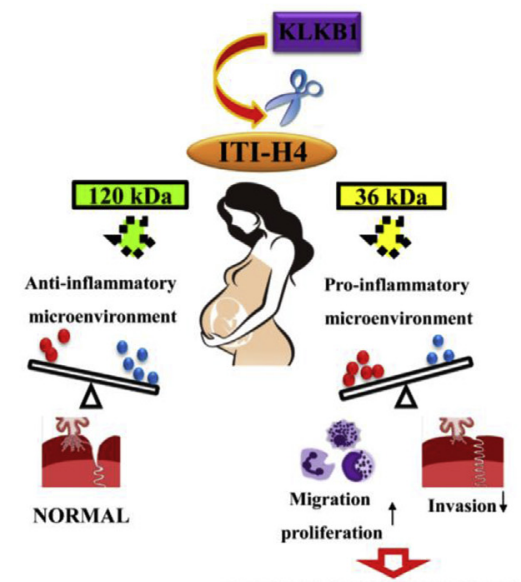

RECURRENT PREGNANCY LOSS

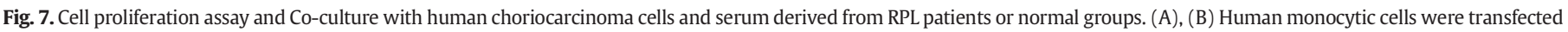

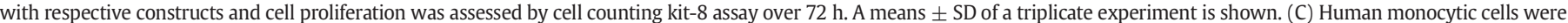

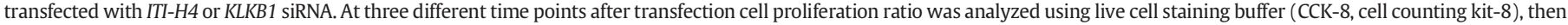

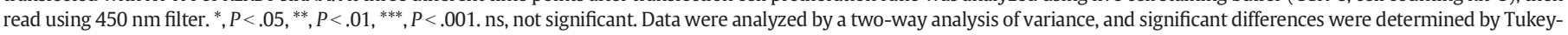

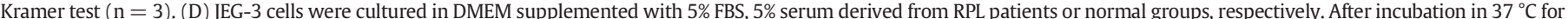

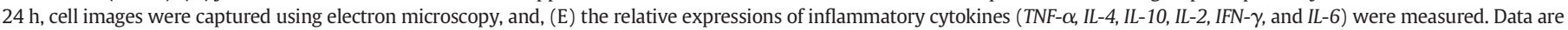

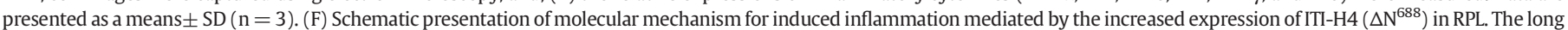

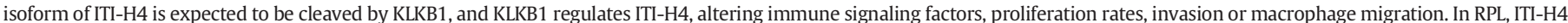

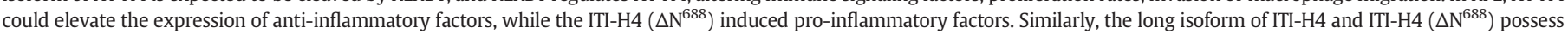
opposing functions on immune tolerance, trophoblast invasion, and monocytes migration or proliferation.

homeostasis in pregnancy, and deregulation of IL-6 pathway showed a vicious circle that might be involved in the pathogenesis of recurrent miscarriage. This is the first study on a novel cellular mechanism underlying RPL. Our present study pointed to the opposing roles of the long isoform of ITI-H4 and ITI-H4 $\left(\Delta \mathrm{N}^{688}\right)$ in modulating inflammatory response, cell invasion, migration or proliferation. And the ITI-H4 $\left(\Delta \mathrm{N}^{688}\right)$ might be a crucial inflammatory factor which contributes to the pathogenesis of RPL.

The Supplementary Material for this article can be found online.

Supplementary data to this article can be found online at https://doi. org/10.1016/j.ebiom.2018.10.029.

\section{Acknowledgements}

We would like to thank members of the Fertility Center and Stem Cell Institute at CHA University and Dr. Hye-Bin Noh and Dr. Sung-Ji Yun at CHA General Hospital for their critical comments on this manuscript.

\section{Funding sources}

This study was supported by the Ministry of Health \& Welfare of the Republic of Korea (grant numbers, HI18C0378) through the Korea Health Industry Development Institute.

\section{Declaration of interests}

none declared.

\section{Author contributions}

L.L. designed and performed experiments, analyzed data and wrote the manuscript; B.C., S.J.S. and C.Z.C. performed the experiments and analyzed the data; K.Y.L., J.P. and J.E.R. analyzed the data and edited the manuscript; K.H.B. conceptualized the research, directed the study and wrote the manuscript.

\section{References}

[1] Lee J, Oh J, Choi E, Park I, Han C, Kim DH, et al. Differentially expressed genes implicated in unexplained recurrent spontaneous abortion. Int J Biochem Cell Biol 2007; 39(12):2265-77.

[2] Chaudhry K, Siccardi M. Blighted Ovum (Anembryonic Pregnancy). StatPearls. Treasure Island FL: StatPearls Publishing LLC; 2018.

[3] Baek KH. Aberrant gene expression associated with recurrent pregnancy loss. Mol Hum Reprod 2004;10(5):291-7.

[4] Caetano R, Ramisetty-Mikler S, Floyd LR, McGrath C. The epidemiology of drinking among women of child-bearing age. Alcohol Clin Exp Res 2006;30(6):1023-30.

[5] Pandey MK, Rani R, Agrawal S. An update in recurrent spontaneous abortion. Arch Gynecol Obstet 2005;272(2):95-108. 
[6] Friis CM, Paasche Roland MC, Godang K, Ueland T, Tanbo T, Bollerslev J, et al. Adiposity-related inflammation: effects of pregnancy. Obesity (Silver Spring) 2013;21(1):E124-30.

[7] Park DW, Yang KM. Hormonal regulation of uterine chemokines and immune cells. Clin Exp Reprod Med 2011;38(4):179-85.

[8] Comba C, Bastu E, Dural O, Yasa C, Keskin G, Ozsurmeli M, et al. Role of inflammatory mediators in patients with recurrent pregnancy loss. Fertil Steril 2015;104(6): 1467-74.

[9] Mor G, Cardenas I, Abrahams V, Guller S. Inflammation and pregnancy: the role of the immune system at the implantation site. Ann N Y Acad Sci 2011;1221:80-7.

[10] Baek KH, Lee EJ, Kim YS. Recurrent pregnancy loss: the key potential mechanisms. Trends Mol Med 2007;13(7):310-7.

[11] Kwak-Kim JY, Chung-Bang HS, Ng SC, Ntrivalas EI, Mangubat CP, Beaman KD, et al. Increased $\mathrm{T}$ helper 1 cytokine responses by circulating $\mathrm{T}$ cells are present in women with recurrent pregnancy losses and in infertile women with multiple implantation failures after IVF. Hum Reprod 2003;18(4):767-73.

[12] Kim MS, Gu BH, Song S, Choi BC, Cha DH, Baek KH. ITI-H4, as a biomarker in the serum of recurrent pregnancy loss (RPL) patients. Mol Biosyst 2011;7(5):1430-40.

[13] Bhanumathy CD, Tang Y, Monga SP, Katuri V, Cox JA, Mishra B, et al. Itih-4, a serine protease inhibitor regulated in interleukin-6-dependent liver formation: role in liver development and regeneration. Dev Dyn 2002;223(1):59-69.

[14] Scheller J, Chalaris A, Schmidt-Arras D, Rose-John S. The pro- and anti-inflammatory properties of the cytokine interleukin-6. Biochim Biophys Acta 2011;1813(5): 878-88.

[15] Teng CB, Diao HL, Ma H, Cong J, Yu H, Ma XH, et al. Signal transducer and activator of transcription 3 (Stat3) expression and activation in rat uterus during early pregnancy. Reproduction 2004;128(2):197-205.

[16] Mohamed E, Jayapalan JJ, Abdul-Rahman PS, Omar SZ, Hashim OH. Enhanced expression of a $35 \mathrm{kDa}$ fragment of inter-alpha-trypsin inhibitor $\mathrm{H} 4$ in sera of healthy pregnant women and patients with hydatidiform mole. Biomark Res 2013;1(1):19.

[17] Diamandis EP, Yousef GM. Human tissue kallikreins: a family of new cancer biomarkers. Clin Chem 2002;48(8):1198-205.

[18] Lim KH, Suresh B, Park JH, Kim YS, Ramakrishna S, Baek KH. Ubiquitin-specific protease 11 functions as a tumor suppressor by modulating Mgl-1 protein to regulate cancer cell growth. Oncotarget 2016;7(12):14441-57.

[19] Gonzalez-Ramon N, Hoebe K, Alava MA, Van Leengoed L, Pineiro M, Carmona S, et al. Pig MAP/ITIH4 and haptoglobin are interleukin-6-dependent acute-phase plasma proteins in porcine primary cultured hepatocytes. Eur J Biochem 2000;267(6): 1878-85.

[20] Bohm D, Keller K, Wehrwein N, Lebrecht A, Schmidt M, Kolbl H, et al. Serum proteome profiling of primary breast cancer indicates a specific biomarker profile. Oncol Rep 2011;26(5):1051-6.

[21] van den Broek I, Sparidans RW, Schellens JH, Beijnen JH. Sensitive liquid chromatography/tandem mass spectrometry assay for absolute quantification of ITIH4-derived putative biomarker peptides in clinical serum samples. Rapid Commun Mass Spectrom 2010;24(13):1842-50.
[22] Chandler KB, Brnakova Z, Sanda M, Wang S, Stalnaker SH, Bridger R, et al. Sitespecific glycan microheterogeneity of inter-alpha-trypsin inhibitor heavy chain H4. J Proteome Res 2014;13(7):3314-29.

[23] Joseph K, Kaplan AP. Formation of bradykinin: a major contributor to the innate inflammatory response. Adv Immunol 2005;86:159-208.

[24] Mejias-Luque R, Peiro S, Vincent A, Van Seuningen I, de Bolos C. IL-6 induces MUC4 expression through gp130/STAT3 pathway in gastric cancer cell lines. Biochim Biophys Acta 2008;1783(10):1728-36.

[25] OTierney-Ginn PF, Lash GE. Beyond pregnancy: modulation of trophoblast invasion and its consequences for fetal growth and long-term children's health. J Reprod Immunol 2014;104-105:37-42.

[26] Rokavec M, Oner MG, Li H, Jackstadt R, Jiang L, Lodygin D, et al. IL-6R/STAT3/miR-34a feedback loop promotes EMT-mediated colorectal cancer invasion and metastasis. J Clin Invest 2014;124(4):1853-67.

[27] Larsen EC, Christiansen OB, Kolte AM, Macklon N. New insights into mechanisms behind miscarriage. BMC Med 2013;11:154.

[28] Zenclussen AC, Fest S, Busse P, Joachim R, Klapp BF, Arck PC. Questioning the Th1/ Th2 paradigm in reproduction: peripheral levels of IL-12 are down-regulated in miscarriage patients. Am J Reprod Immunol 2002;48(4):245-51.

[29] Kwak-Kim J, Park JC, Ahn HK, Kim JW, Gilman-Sachs A. Immunological modes of pregnancy loss. Am J Reprod Immunol 2010;63(6):611-23.

[30] Blom AM, Morgelin M, Oyen M, Jarvet J, Fries E. Structural characterization of interalpha-inhibitor. Evidence for an extended shape. J Biol Chem 1999;274(1):298-304.

[31] Salier JP, Rouet P, Raguenez G, Daveau M. The inter-alpha-inhibitor family: from structure to regulation. Biochem J 1996;315(Pt 1):1-9.

[32] Nishimura H, Kakizaki I, Muta T, Sasaki N, Pu PX, Yamashita T, et al. cDNA and deduced amino acid sequence of human PK-120, a plasma kallikrein-sensitive glycoprotein. FEBS Lett 1995;357(2):207-11.

[33] Song J, Patel M, Rosenzweig CN, Chan-Li Y, Sokoll LJ, Fung ET, et al. Quantification of fragments of human serum inter-alpha-trypsin inhibitor heavy chain 4 by a surfaceenhanced laser desorption/ionization-based immunoassay. Clin Chem 2006;52(6): 1045-53.

[34] Prins JR, Gomez-Lopez N, Robertson SA. Interleukin-6 in pregnancy and gestational disorders. J Reprod Immunol 2012;95(1-2):1-14.

[35] Jovanovic M, Vicovac L. Interleukin-6 stimulates cell migration, invasion and integrin expression in HTR-8/SVneo cell line. Placenta 2009;30(4):320-8.

[36] Rezaei A, Dabbagh A. T-helper (1) cytokines increase during early pregnancy in women with a history of recurrent spontaneous abortion. Med Sci Monit 2002;8 (8):CR601-610.

[37] Singh H, Aplin JD. Adhesion molecules in endometrial epithelium: tissue integrity and embryo implantation. J Anat 2009;215(1):3-13.

[38] Xu B, Sun X, Li L, Wu L, Zhang A, Feng Y. Pinopodes, leukemia inhibitory factor, integrin-beta3, and mucin-1 expression in the peri-implantation endometrium of women with unexplained recurrent pregnancy loss. Fertil Steril 2012;98(2):389-95.

[39] Balasubramanian D, Harper L, Shopsin B, Torres VJ. Staphylococcus aureus pathogenesis in diverse host environments. Pathog Dis 2017:75(1). 